\title{
Design Analysis of Vertical Axis Wind Turbine Blade Using Biomimicry
}

\author{
Vemuluri Prathik, Udith Kumar Narayanan and Pankaj Kumar
}

\author{
SRM institute of Science and Technology, Kattankulathur, Tamil Nadu, India
}

\begin{abstract}
Performance and efficiency of a Vertical Axis Wind Turbine improved by using a modified turbine blade, derived from biological features, by harness more wind energy. The detailed simulations were carried in Q-blade's X-foil, Java foil and Sim Scale software at low wind speed with bio-mimicable cambered foil add-ons to different biostructure blades such as Maple seed leaf, Eagle wing. Among these simulations, the Corrugated Dragonfly vein FX 63-137 foil shows improved performance over cambered foils and FX 63-37 itself. The Maple-Wing combined blade structure showed an improved lift-drag ratio with a high coefficient of power.
\end{abstract}

Keywords: Vertical Axis Wind Turbine (VAWT), Tip Speed Ratio (TSR), Vorticity, Lift coefficient $\left(\mathrm{C}_{\mathrm{L}}\right)$, Drag coefficient $\left(C_{D}\right)$ Power coefficient $\left(C_{P}\right)$.

\section{INTRODUCTION}

Wind energy is only limited by the technology which in the past have seen phenomenal improvements in Horizontal wind turbine systems, yet they still incur huge installation costs and massive sizes to maintain good power generation, that ordinary citizens cannot usually consider utilizing. To enable access of wind energy to the common, the Vertical axis wind turbines (VAWT) have been of vital importance in changing the dynamics of wind energy designing. Their scalable sizes and low-cost installation were found beneficial for usage in a wide range of wind speeds, but the drawbacks are the lower efficiency, which generally needs external devices such as vortex struts, fins, motors and various mechanisms that in the long run inhibits the performance and cannot extract proper speeds for necessary power generation.

To begin, first detailed information of important aspects of VAWT (Blade aerofoil, Reynolds number, Strut design, Solidity, Number of blades, Blade-strut, connection point, Blade pitch angle, Height-to-radius ratio, Blade aspect ratio, Chord-to-radius ratio, Blade taper, Blade orientation, Blade coning angle etc), a review paper by Hand et al. [1] which gave importance to parameters like power output, efficiency, optimum tip-speed ratio and operational range. Also, it was discussed how a VAWT's performance is sensitive to drag losses, especially at a high Tip Speed Ratio (TSR).

\footnotetext{
*Address correspondence to this author at the Dept. of Mechanical Engineering, SRMIST Kattankulathur, Chennai, India;

E-mail; pankajkr@srmist.edu.in
}

To accommodate losses, the blade struts must have a streamlined profile section to minimize the impact of the parasitic torque. Darrieus VAWT for power generation and configurations were explained for maximum efficacy [2].

Battisti et al. [3] conducted an aerodynamic analysis of Darrieus for different architectures such as $\mathrm{H}$ type, Giro, Helical etc. for optimum TSR and performance using blade element momentum model for tip vorticity and Prandtl correction. Helical seemed effective but complex to build whereas the straight blade $\mathrm{H}$ rotor was simpler and equally efficient considering power losses and neglecting support structures. In the similar study, analysis was done to determine the self-start capabilities, lift, stall and drag properties from National Advisory Committee for Aeronautics (NACA) foils. It was found that symmetric foils are not suitable for small capacity VAWT and require low drag asymmetric thick foils [4]. In the line of improvement of power output, researchers found that with increase in number of blades there is an increment in power. But there is another factor which comes into play: pulsating power which is not efficient. They used an experimental setup where the blades are interchangeable and found that four blades have lower power output than expected [5]. Cai et al. [6] found that the aerodynamic performance of the near region of blade tips is improved with Winglets which increases the overall torque. But winglets do not improve it at every azimuth angle for each blade.

There are few studies related to Bio-Inspired corrugated aerofoils for the higher performance. In the VAWT, the tubercles made it structurally more stable, but the samara and maple showed similar behaviour in terms of lift and drag to the FX blade [7]. Study of 
golden eagle wing geometry with secondary wing for aerodynamics was analysed in fluent. It fit the steady flow conditions with performance improvements at high pitch angles using secondary wing structure [8]. Flexible blade structure made of bamboo laminate with dragonfly vein corrugated add on was simulated and it gave the advantage of delayed stall and better adaptation over similar base foil structure [9].

Meana-Fernández et al. [10] presented numerical work in the paper where they explained the important factors necessary to consider using JAVA foil. There are various numerical studies reported for the vertical axis wind turbine which explains the coherent flow structures near blade accurately. These simulations help us to understand the way to improve the efficiency of a turbine by analysing the design parameters. Element Momentum theory (BEM) is such a method adopted for simulating flow around blades with the help of Q-Blade. One of the earlier studies, NACA 0018 aerofoil was analysed by using Q-Blade accurately. The blade performance explained with help of XFOIL and Q-Blade accurately. The performance of different aerofoils at different operational loads. The analysis of (NACA 2412) and (SG 6042) aerofoils are done and compared by using Q-Blade software, Alaskari et al. [11] have done the design and optimization analysis of SG6043 aerofoil with Q-Blade software and reported that Q-Blade software is reliable to analyse the blades of wind turbines. We need to understand the formulas which govern them and formulas which define the characteristic feature of wind turbines. After understanding the characters of the wind turbine research was done on commercially used wind turbine and looked into the aspects needed to improve $[12,13]$ while designing aerofoil places improvements were made as suggested by Anand et al. [14].

The purpose of this paper is to improve the performance of Vertical Axis wind turbine blade designs by lift-drag modulations, our design approach involves accommodating a new VAWT blade design adopted from the specimens using the concept of biomimicable. Here, we attempt to simplify the blade design by addressing the unexplored capabilities of the specimen features through the utilization of the different aerofoil-blade structural. By blade designing, the implementation is by different combination in its various design ratios, such that each feature added plays a role in balancing a ratio of maximum torque, power and better wind speed ranges with minimum stresses induced. To work with the foil-blades, a small scalable Darrieus rotor models were designed based on height-radius ratios and chord length for the simulations which is carried out by using Q-blade's Xfoil and Java foil and later simulated with bio-mimicable add-ons in Sim Scale software for different NACA and Cambered foils, different blade modules of the foil with bio structures of specimens like Maple seed leaf, Eagle wing were further simulated in Q-blade. The design parameters (Calculating $\mathrm{C}_{\mathrm{p}}, \mathrm{C}_{\mathrm{l}}, \mathrm{C}_{\mathrm{d}}, \mathrm{Cm}, \mathrm{TSR}$ ) are evaluated at a low range of speed based on suitability for urban areas.

\section{THEORETICAL FORMULATION}

The design parameters used to analyse in Q-blade, include $C_{l}, C_{d}, C_{p}, C_{m}$ and TSR which are explained in detail below.

Lift Coefficient $\left(C_{\mathrm{L}}\right)$ - It expresses the ratio of the lift force to the force produced by the dynamic pressure times the area. It is evaluated here as $C_{\mid}$where $v$ is velocity of wind, $A$ is the area of blade, $\rho$ is the density of wind and $F_{L}$ is the lift force generated along lift angle -

$$
C_{L}=\frac{2 F_{l}}{\rho A v^{2}}
$$

Drag Coefficient $\left(C_{D}\right)$ - It expresses the ratio of the drag force to the force produced by the dynamic pressure times the area. It is evaluated as $C_{d}$ where $F_{D}$ is drag force generated along lift angle -

$C_{D}=\frac{2 F_{d}}{\rho A V^{2}}$

Power Coefficient $\left(\mathbf{C}_{p}\right)$ - The power coefficient explain that how efficiently a turbine converts the energy in the wind to electricity. It is evaluated here as $C_{p}$ where $v_{1}$ is the initial wind velocity $v_{2}$ is the final wind velocity -

$C_{p}=\frac{\rho A\left(V_{1}^{2}-V_{2}^{2}\right)\left(V_{1}+V_{2}\right)}{2 \rho A V_{1}^{2}}$

Torque Coefficient $\left(\mathbf{C}_{\mathrm{m}}\right)$ - It expresses the ratio of the rotational torque to the torque produced by the wind speed alone. It is evaluated here as $C_{m}$ where $v$ is the velocity of wind -

$C_{m}=\frac{F_{l-} F_{d}}{\rho A v^{2}}$

Coefficient of Pressure (C. $\left.\mathbf{p}^{*}\right)$ - It denotes the relative pressure ratio near the vicinity of the foil 
compared to the free stream wind from the wind tunnel. It is evaluated as $\mathrm{Cp}^{*}$ where $\mathrm{P}$ is pressure near the foil and $P_{\infty}$ is the pressure infinite distance away from the foil and $v$ is wind velocity-

$$
C_{p} *=\frac{P-P_{\infty}}{2 \rho v}
$$

Tip Speed Ratio (TSR) - It is the ratio between the tangential speed of the tip of a blade and the actual speed of the wind. It is evaluated here as $\lambda$ where $\omega$ is angular velocity of blade and $R$ is the radius of rotor turbine-

$$
\lambda=\frac{\omega R}{v}
$$

\section{METHODOLOGY}

First, the foils NACA 0012, NACA 0015, NACA 0018, NACA 0022, NACA 4312 and NACA 4412 were selected. Asymmetric or non-cambered foils S809, S1012, S1210, DU 06 W200, FX 63 137, UO 17 LDA were chosen based on supporting VAWT blades $[8,10]$. Q-Blade was used to analyse the foils to find the best suitable and efficient foil structure under different wind conditions based on torque, lift and drag ratios. For which Q-Blade's X-Foil was used, the conditions of the new polar simulation were selected under X-direct Tab where the Reynolds Number and Mach numbers were selected based on average wind conditions of Chennai city. The best performing foils were further improved and interpolated using foil optimization tool available within Q-Blade and then compared based on pressure coefficient using Sim Scale CFD and Java-foil governed by a Turbulent Kinetic energy model $(k)$ and the optimum performance foil based on the conditions was selected. Secondly, Bio-features suggested from the literature review were added to the foil basis selected among the previous foils. The addition of the bio-features involved an embedded interpolation technique available within QBlade that adjusts the surface co-ordinate points in proportion to reference image along with manual adjustment of foil points scaled. In similar way to previous steps, further optimum performing foil among the bio-foil designs has been made. Futrther, a small scalable blade architecture and model is required with a simplified design to begin further blade simulations, for which straight blade Darrieus with two strut bars on each blade attached and supporting to the rotor shaft seemed ideal, since the minimum no. of 2 struts provided more motion while also maintaining rigidity [7].
Finally, different straight blades were modified with overall structural and gradual changes, incorporating additional bio features such that each modification subsequently provided step by step improvement over the straight blade. All identical structural changes were compared using DMS and LLT simulations, the changes include overall structural changes, sectional modifications along the blade length, bio-additions and add-ons (section 4.4). DMS or [11] Double Multiple Stream-tube is an embedded method within Q-Blade that uses virtual stream tubes which are split up into smaller flow tubes and during $360^{\circ}$ path simulation the turbine blades pass through these tubes, the energy extracted and velocity reduction of the fluid is compared, The DMS rotor and turbine methods uses this to compare the power coefficient, tip speed ratios of the blade, velocity and rotor power.

LLT or Non-linear laminar turbulent simulation model is another embedded Q-Blade method used for generating wind flow field-based simulation to detect flow distribution and vortices generated from the turbine [11]. For the setup, a 1.5 meter height, 2 meter width and 50 meter length cylindrical mesh domain with an inlet wind field plane perpendicular to the rotor was used, operational points of TSR was selected at 4 and an average RPM of 300 was set based on the regional average wind speeds found as well as the results from $X$-Foil, The model is kept at an azimuthal angle of $270^{\circ}$ to ensure faster initial cut-in. The inflow speeds are kept at maximum $12 \mathrm{~m} / \mathrm{s}$ speeds with zero horizontal inflow, the ground effects are kept negligible with power law-based wind shear profile, the computation is for 3 seconds with 300 -time steps, each time step is 0.02 seconds long.

\section{RESULTS}

\subsection{Base Foil Simulations}

The NACA prefix foils mentioned have a cambered structure with symmetry on both pressure surfaces. The predefined foil coordinates of each NACA foil mentioned above were already embedded in the $Q$ blade software. So, each NACA was imported from the predefined file under the foil menu. NACA 0012, 0015, 0018, 0022 are all having zero camber and have a double symmetric cross section about the same horizontal centre line. Where as NACA 4312 and 4416 have a $4 \%$ camber and have a distinct centre line.

Foils were simulated under the XFOIL analysis tab for each foil. The set conditions of the new polar simulation were selected under $\mathrm{X}$-direct Tab where the 
Table 1: Values from XFOIL Simulation of NACA Foils from Q-Blade, Comparing Lift Coefficient $\left(C_{l}\right)$, Drag Coefficient $\left(C_{m}\right)$, Peak Tip Speed and Lift by Drag Ratio with Corresponding Angle of Attack

\begin{tabular}{|c|c|c|c|c|c|c|}
\hline Symmetric Foils & $\begin{array}{c}\text { NACA } \\
0012\end{array}$ & $\begin{array}{c}\text { NACA } \\
0015\end{array}$ & $\begin{array}{c}\text { NACA } \\
0018\end{array}$ & $\begin{array}{c}\text { NACA } \\
0022\end{array}$ & $\begin{array}{c}\text { NACA } \\
4312\end{array}$ & $\begin{array}{c}\text { NACA } \\
4416\end{array}$ \\
\hline $\mathrm{C}_{\mathrm{I}}$ & $\begin{array}{c}\alpha=10.5^{\circ} \\
0.972\end{array}$ & $\begin{array}{c}\alpha=11.5^{\circ} \\
1.062\end{array}$ & $\alpha=12.5^{\circ} 1.133$ & $\begin{array}{c}\alpha=11^{\circ} \\
1.133\end{array}$ & $\begin{array}{c}\alpha=11.5^{\circ} \\
1.491\end{array}$ & $\begin{array}{c}\alpha=13^{\circ} \\
1.478\end{array}$ \\
\hline $\mathbf{C}_{\mathrm{m}}$ & $\begin{array}{l}\alpha=11^{\circ} \\
0.029\end{array}$ & $\begin{array}{l}\alpha=13^{\circ} \\
0.043\end{array}$ & $\begin{array}{c}\alpha=14.5^{\circ} \\
0.051\end{array}$ & $\begin{array}{c}\alpha=14^{\circ} \\
0.055\end{array}$ & $\begin{array}{l}\alpha=16^{\circ} \\
-0.023\end{array}$ & $\begin{array}{l}\alpha=17^{\circ} \\
-0.023\end{array}$ \\
\hline L/D & $\begin{array}{c}\alpha=5^{\circ} \\
36.609\end{array}$ & $\begin{array}{c}\alpha=6^{\circ} \\
37.429\end{array}$ & $\begin{array}{l}\alpha=6.5^{\circ} \\
37.250\end{array}$ & $\begin{array}{c}\alpha=8^{\circ} \\
35.693\end{array}$ & $\begin{array}{l}\alpha=11^{\circ} \\
46.511\end{array}$ & $\begin{array}{c}\alpha=10.5^{\circ} \\
44.513\end{array}$ \\
\hline High tip speed & $\alpha=10.5^{\circ}$ & $\alpha=12^{\circ}$ & $\alpha=14.5^{\circ}$ & $\alpha=13.5^{\circ}$ & $\alpha=17.5^{\circ}$ & $\alpha=17^{\circ}$ \\
\hline
\end{tabular}

Reynolds Number and Mach numbers were required. For low velocity conditions required average turbulent wind conditions, Therefore Reynolds number was chosen at 100,000 . The peak values of noticeable coefficients were compared and tabulated in [Table 1].

(a)

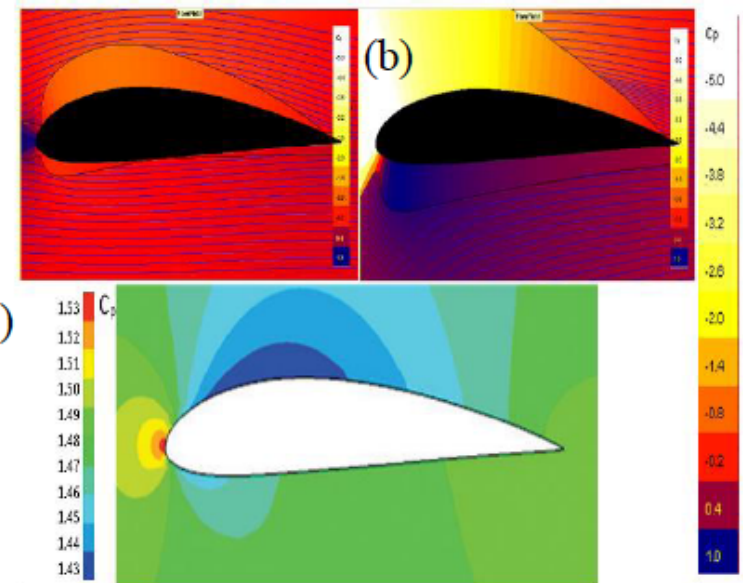

Figure 1: Pressure distribution ( $\left.\mathrm{Cp}^{*}\right)$ for NACA 4416 (a) zero angle, (b) $17^{\circ}$ angle of attack) using java foil compared with (c) pressure distribution at $0^{\circ}$ [14].
Asymmetric foils have proven to provide better capacity in producing constant torque at fluctuating angle of attack [4]. FX 63-137 and UO 17 LDA [14, 10] have also surpassed most of the efficient NACA blades at equivalent varying angles according to simulations [12] with a close characteristic with the NACA 4416 foil as shown in Figure 1 and Table 1.

The other asymmetric foils chosen were also compared through the same simulations under XFOIL analysis for the same conditions of Reynolds number 100,000 shown in [Figure 2] and the maximum potential values were compared [Table 2].

FX 63-137 has shown an increase in coefficient of pressure $\left(\mathrm{Cp}^{*}\right)$ making it a viable foil structure basis for an overall high performance VAWT design. This is also evident from the research papers on improving efficiency UO 17 LDA has proved to be a competitor to FX 63-137 [14]. Hence, they were compared through flow field simulations [Figures 3 and 4]. Due to similarity in NACA 4416 [3] and FX 63-137 they were further compared through simulations for suitable base

Table 2: Values from XFOIL Simulation of Cambered foils from Q-Blade, Comparing Lift Coefficient $\left(C_{1}\right)$, Drag Coefficient $\left(\mathrm{C}_{\mathrm{m}}\right)$, Peak tip Speed and Lift by Drag Ratio with Corresponding Angle of Attack

\begin{tabular}{|c|c|c|c|c|c|c|}
\hline Asymmetric Cambered Foils & S809 & S1012 & $\mathrm{S} 1210$ & FX 63-137 & DU 06-W200 & UO 17 LDA \\
\hline $\mathrm{Cl}$ & $\begin{array}{l}\alpha=24^{\circ} \\
0.801\end{array}$ & $\begin{array}{c}\alpha=24.5^{\circ} \\
0.801\end{array}$ & $\begin{array}{c}\alpha=19.5^{\circ} \\
1.258\end{array}$ & $\begin{array}{c}\alpha=19.5^{\circ} \\
1.199\end{array}$ & $\begin{array}{l}\alpha=33^{\circ} \\
1.140\end{array}$ & $\begin{array}{c}\alpha=22^{\circ} \\
0.885\end{array}$ \\
\hline $\mathrm{Cm}$ & $\begin{array}{c}\alpha=10.5^{\circ} \\
0.054\end{array}$ & $\begin{array}{l}\alpha=5^{\circ} \\
0.038\end{array}$ & $\begin{array}{c}\alpha=2^{\circ} \\
-0.072\end{array}$ & $\begin{array}{c}\alpha=-3.5^{\circ} \\
0.011\end{array}$ & $\begin{array}{l}\alpha=7^{\circ} \\
0.034\end{array}$ & $\begin{array}{l}\alpha=5^{\circ} \\
0.018\end{array}$ \\
\hline L/D & $\begin{array}{c}\alpha=19.5^{\circ} \\
2.536\end{array}$ & $\begin{array}{c}\alpha=6^{\circ} \\
8.622\end{array}$ & $\begin{array}{c}\alpha=5.5^{\circ} \\
6.202\end{array}$ & $\begin{array}{l}\alpha=6.5^{\circ} \\
5.320\end{array}$ & $\begin{array}{c}\alpha=33^{\circ} \\
3.04\end{array}$ & $\begin{array}{l}\alpha=16^{\circ} \\
2.955\end{array}$ \\
\hline Angle of high $Q$ speed & $\begin{array}{c}\alpha=21^{\circ} \\
1.65\end{array}$ & $\begin{array}{c}\alpha=22^{\circ} \\
1.57\end{array}$ & $\begin{array}{c}\alpha=20.5^{\circ} \\
1.67\end{array}$ & $\begin{array}{c}\alpha=21.5^{\circ} \\
1.64\end{array}$ & $\begin{array}{c}\alpha=34^{\circ} \\
1.73\end{array}$ & $\begin{array}{c}\alpha=22^{\circ} \\
1.53\end{array}$ \\
\hline
\end{tabular}


foil [Figures 1 and 4]. The FX 63-137 foil seemed best for our further VAWT blade design improvement analysis (see discussion section 5.1).

(a)

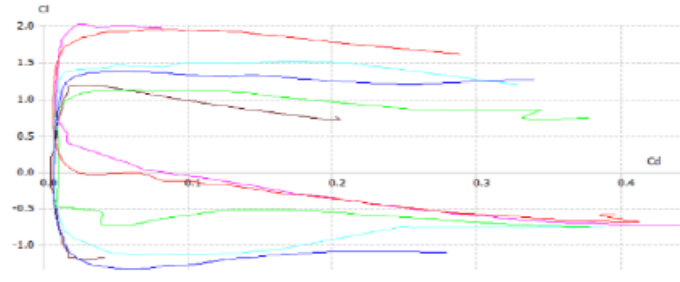

(b)

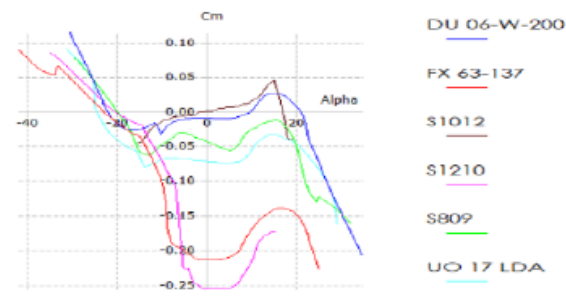

Figure 2: XFOIL simulations of cambered foils Comparison between (a) $\mathrm{Cl}$ vs $\mathrm{Cd}$, (b) $\mathrm{Cm}$ at different angles.

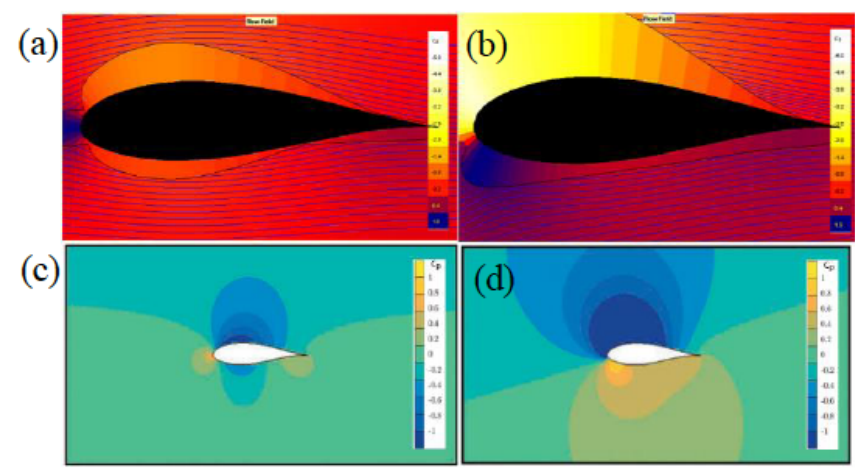

Figure 3: Pressure distribution for UO 17 LDA (a) -zero angle of attack, (b)- $11.5^{\circ}$ angle of attack using java foil compared with reference $[11](\mathbf{c}) \&(\mathbf{d})$.

(a)

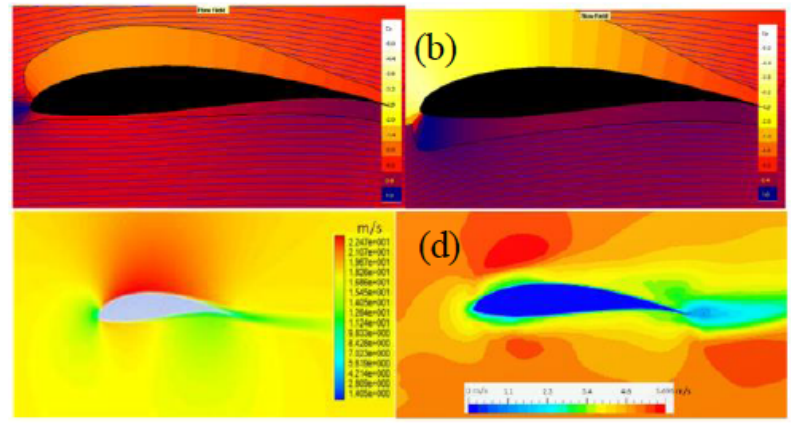

Figure 4: Pressure distribution for FX 63137 (a)-zero angle of attack, (b) $8.5^{\circ}$ angle of attack) using java foil (c) $0^{\circ}$ reference [3] and (d) velocity distributions by sim scale CFD.

\subsection{Modified Bio Mimicked Foils}

Bio-mimicable features with aerodynamic capabilities worth incorporating into the dynamic VAWT blades using base foil FX $63-137$ as the standard reference foil included the Golden Eagle wing, Dragon Fly wing, Maple seed leaf. Whereas the Golden Eagle wing structure and [7-9] Dragonfly vein structure are quite suitable for the sizable blade cross section, hence their design features were suitable for adding on the base foil [Figures 5, 6 and 7]. The corrugated pattern of the veins on dragon fly's transparent wings enables dragon flies to maintain fixed altitudes and swift flight trajectories for long durations of time without intervals helping them migrate great distances in short time periods.

A full-grown golden eagle wingspan around a meter, the feathers in each wing are rigid yet lightweight, promoting the eagles to manoeuvre at high speeds without losing those feathers, the cross section of its wing contains a set of feathers, a main feather and a secondary feather.

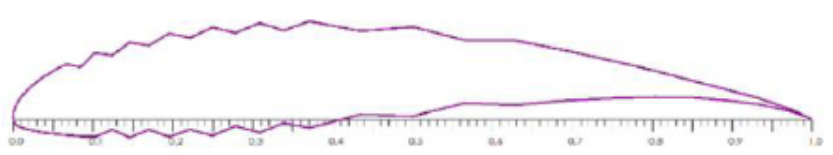

Figure 5: Corrugated Foil - based on Dragonfly Vein structure.

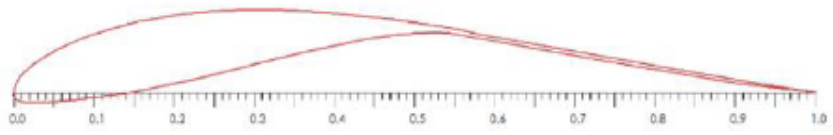

Figure 6: Secondary Tail Foil - based on Golden Eagle Feather structure.

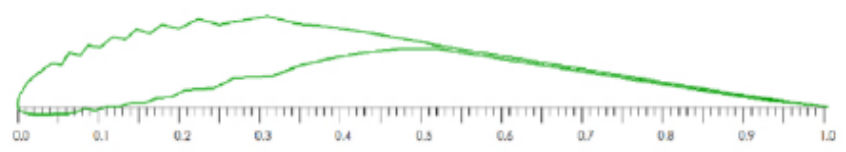

Figure 7: Modified Combined Foil - Combination of Secondary Tail Foil and Corrugated Dragonfly Foil.

XFOIL analysis on the three modified FX 63 137s were conducted in $Q$ blade to find the profile lift and drag conditions and analyse the differences. Relations such as the drag-lift, power coefficient and torque coefficient were found and shown in [Figure 8] and the peak values were also noted for required comparison of efficiency [Table 3]. 


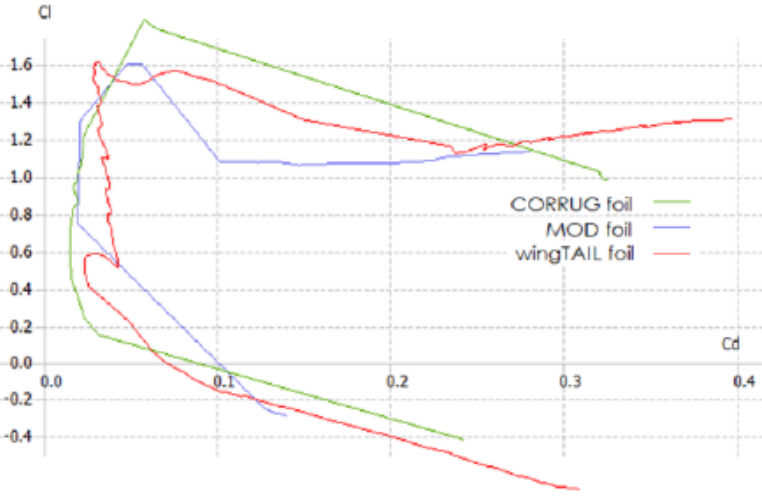

Figure 8: XFOIL simulation graph of coefficient of lift vs coefficient of drag of the different foils.

Simulating the wind trail and vortex of the turbine blades at the start of the wind tunnel to check for starting capabilities. The foils during revolution change angles continuously, the chamber of FX foil over with the bio-features need to impart the necessary torque initiating the rotation which needs to be tested through the nonlinear model (LLT) 3D simulation [Table 4].

Table 3: Values from XFOIL Simulation of Modified Foils from Q-Blade, Comparing Lift Coefficient $\left(C_{1}\right)$, Drag Coefficient $\left(C_{d}\right)$ with Angle of Attacks at their Peak Lift by Drag Ratios (L/D)

\begin{tabular}{|c|c|c|c|c|}
\hline $\begin{array}{c}\text { Chosen and } \\
\text { Modified Foils }\end{array}$ & $\begin{array}{c}\text { Angle of } \\
\text { Attack }\end{array}$ & $\mathbf{C}_{\mathbf{l}}$ & $\mathbf{C}_{\mathrm{d}}$ & L/D \\
\hline \hline FX 63-137 Foil & $8.5^{\circ}$ & 1.645 & 0.029 & 56.9 \\
\hline Wing tail Foil & $10^{\circ}$ & 1.55 & 0.029 & 55.3 \\
\hline $\begin{array}{c}\text { Corrug-wing } \\
\text { modified Foil }\end{array}$ & $8^{\circ}$ & 1.3 & 0.0216 & 60.1 \\
\hline Corrugated Foil & $12.6^{\circ}$ & 1.818 & 0.0204 & 64.8 \\
\hline
\end{tabular}

Table 4: Average Values from Nonlinear Lifting Line 3-d Simulations

\begin{tabular}{|c|c|c|c|}
\hline $\begin{array}{c}\text { LLT Foil } \\
\text { Simulation }\end{array}$ & $\mathbf{C}_{\mathrm{m}}$ & Average $\mathrm{C}_{\mathrm{p}}$ & $\begin{array}{c}\text { Average } \\
\text { Power (W) }\end{array}$ \\
\hline \hline FX foil & -0.170 & -0.577 & -1.2155 \\
\hline Wing foil & -0.095 & -0.0114 & -0.0249 \\
\hline $\begin{array}{c}\text { Corrug-wing } \\
\text { modified foil }\end{array}$ & -0.085 & 0.0813 & 0.172 \\
\hline Corrugated foil & -0.144 & 0.3185 & 0.674 \\
\hline
\end{tabular}

\subsection{Optimization of Aspect Ratio and Chord Length}

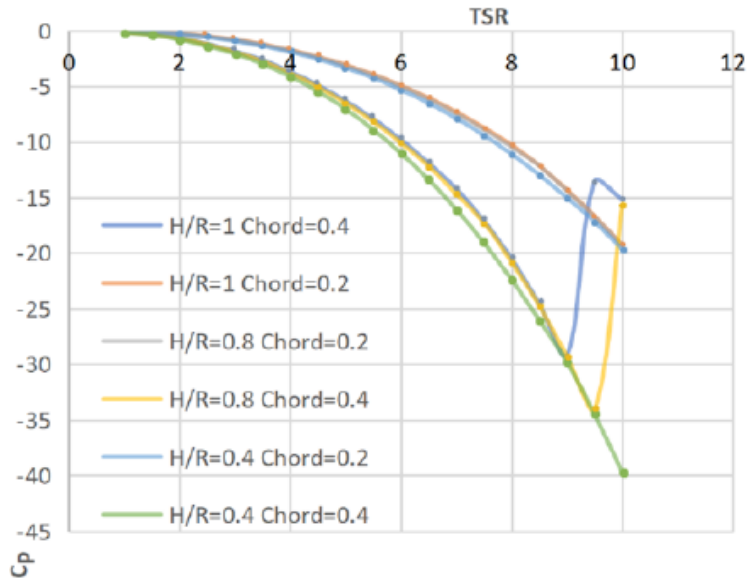

Figure 9: Coefficient of power $\left(C_{p}\right)$ vs TSR of different H/R (height/radius) ratio and chord lengths (metre).

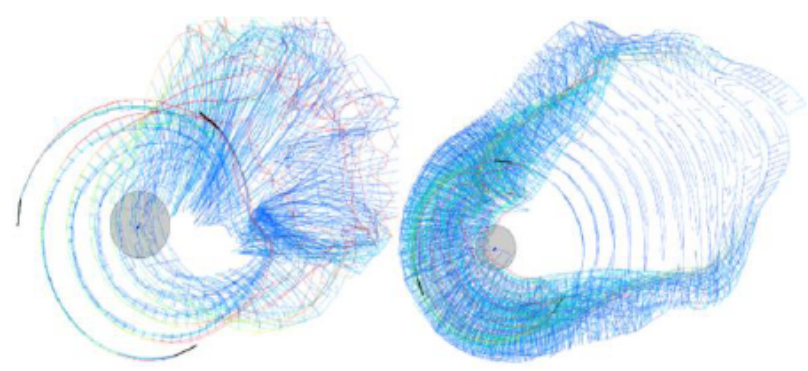

Figure 10: Top views of outward facing blade wind field vorticity (left) and inward facing blade (right) wind field vorticity.

Based on the law of diminishing return the ideal number of blades for tall blade VAWTS is three blade straight blade structure is simulated at different aspect ratios and chord lengths using rotor DMS simulation for low wind speed of $5 \mathrm{~m} / \mathrm{s}$ as shown in [Figure 9] The cutin wind speed was set at $5 \mathrm{~m} / \mathrm{s}$ and cut-out wind speed was set to $12 \mathrm{~m} / \mathrm{s}$ under rotor DMS parameters. The foil position and orientation in the VAWT blade plays an important role in acquiring the efficiency, the blade can be fitted facing inward as well as outward to find a suitable configuration in better acquired wind and blade motion. For the specification requirements of the VAWT to find the right blade orientation two blades of 1 metre hub height, one inward facing and one outward facing were simulated using LLT [Figure 10] and rotor and turbine DMS simulations.

From the rotor DMS obtained the $C_{p}$ vs TSR comparison for the previous wind parameters [Figure 11] the rise in the power coefficient is visible from TSR 3 to 6 for the inward facing blade compared to outward facing blade. In the turbine DMS the stream 


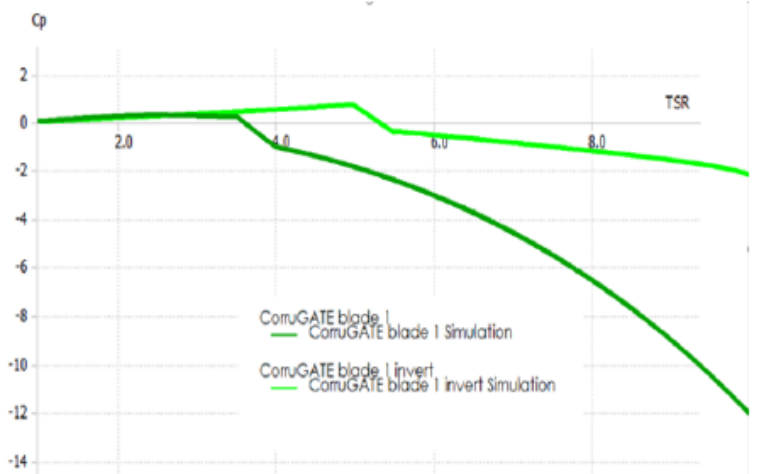

Figure 11: $C_{p}$ vs TSR of inward (shown as invert) and outward facing blade from rotor DMS.

tubes are split up into smaller flow tubes and during $360^{\circ}$ path simulation the turbine blades pass through these tubes, the energy extracted and velocity reduction of the fluid is compared. The turbine DMS of the inward facing blade and outward facing blade from the Velocity vs Power graph [Figure 12] the inward facing was acquiring more power at low velocity winds compared to outward facing blade.

\subsection{Optimum Blade Design Simulations}

For the final blade design, approach was to provide a changing cross sectional chord length such that the design will eventually build itself a non-uniformity similar to the average specimen body shape making it easy for modifying or adding the Bio-mimicked features over it, which would require more trimming for a simple straight blade, working with the varying cross sections also help understand the effects of chord length modifications along the different sections in a sequential manner. So, based on the two-strut-perblade rotor model (discussed in Methodology section $3)$,[1] the centre and edge seemed like the ideal blade section for attempting chord length modifications without hindering the blade strength, since each strut sat between the centre and edge, they were the farthest points away from the point of strut-blade contact along the blade. Therefore, a narrowed centre and a narrowed centre with narrow edge blade [Figure 15(a)] of chord length narrow-to-broad ratio of 1:2 (ratio derived from half Maple leaf referred from [7]) was designed with the selected Corrugated FX 63-137 foil and analysed using LLT (Non-linear laminar turbulent simulation model) at $5 \mathrm{~m} / \mathrm{s}$ wind speed.

The narrow section increased air vorticity towards the edges of the blade with high tip vortex. The thinedge bowtie blade reduced the tip vortices [Figure 13] from red-green-blue contours showing descending air strength with gradient flow from stronger to weaker vortices. The rotor DMS of the narrow blade and bowtie blade gave the $C_{p}$ vs TSR graph which shows that the bowtie efficiency was slightly higher at TSR equal to 5 [Figure 14]. The previously discussed Maple seed leaf and golden eagle features were chosen for the bladeform modification. Based on the back edge narrow centre blade as basis, the bio features included Maple leaf edge and wing edge.

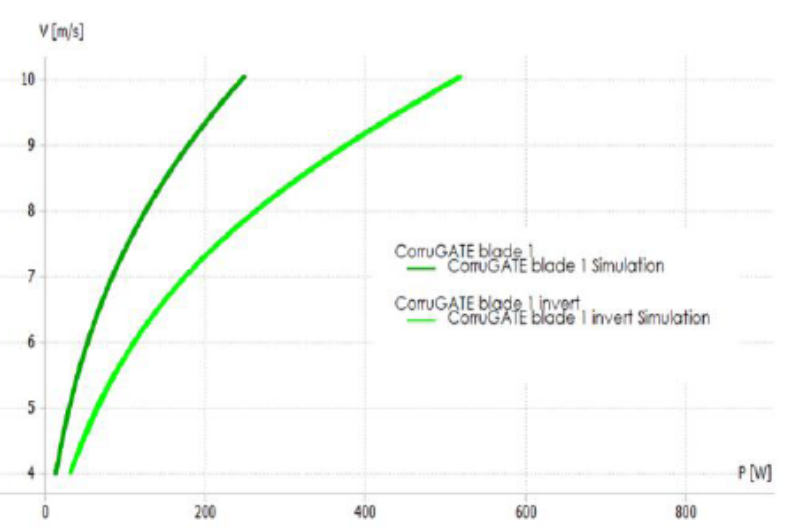

Figure 12: Velocity vs Power of inward (shown as invert) and outward facing blade from turbine DMS.

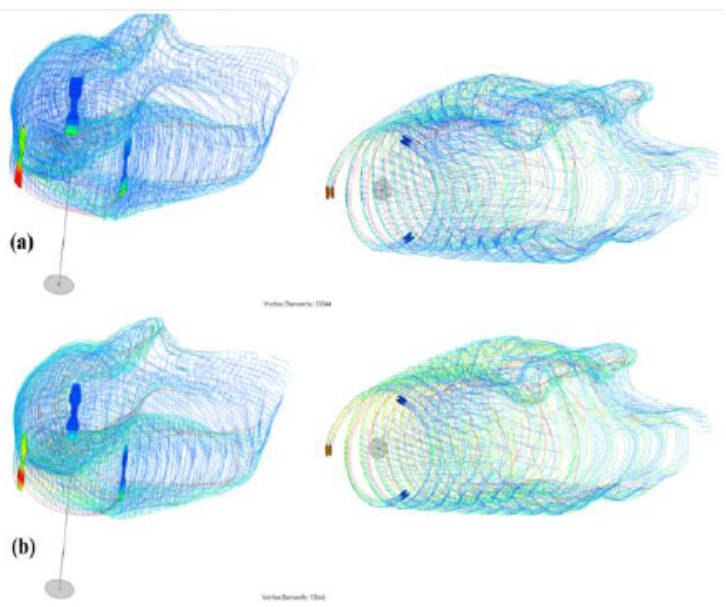

Figure 13: LLT wind field vorticity simulation with blade stress indication of narrow centre (a) and bowtie blade (b) isometric view (left) and top view (right).

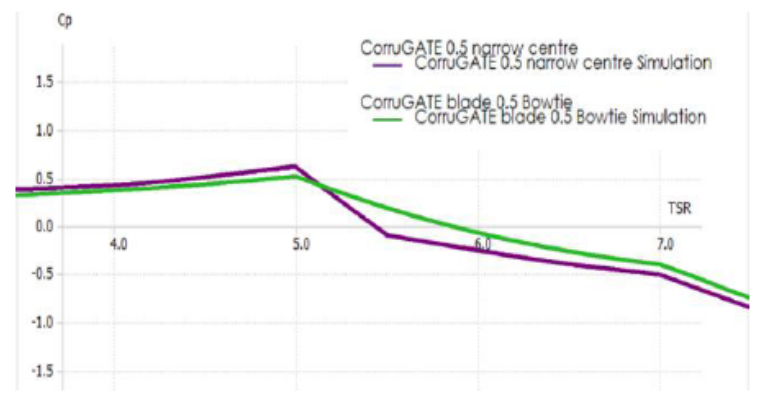

Figure 14: $C_{p}$ vs TSR of narrow centre blade and Bowtie blade. 
The main bio features of Maple leaf and wing tip were directly added over the narrow centre-edge blade (also called Bowtie blade) design. The chord length was modified between the centre and edge [shown in Figure 15 (b) (c)] The Wing blade had a basic structure of the golden eagle wing wingspan with minimum curvature [8]. Maple leaf blade and Wing blade were separately compared among each other through DMS along with the Bowtie blade. [Figure 15] shows the comparison of the rotational torque at different angle of attacks of the main bio features which shows maple seed blade (green curve) and wing blade (red curve) with better torque produced at optimum angle of attack compared to bowtie blade (blue curve) found using turbine DMS.

Maple-wing combination blades with different ratios were designed and a suitable blade among them was incorporated with thin back winglets [6] All introduced and modified design blades of Maple, Wing and Bowtie blades along with the Maple-wing combinations and winglet add-on blades were compared for $C_{p}$ vs TSR using rotor DMS and shown in [Figure 16]. The $C_{p}$ values of bowtie deviated little higher at TSR of 4 , the winglets also provided an increase in $\mathrm{C}_{\mathrm{p}}$ of the Maplewing2 combination blade [Figure 16].

Through LLT rotor simulations show the flow structures of the blades by using $5 \mathrm{~m} / \mathrm{s}$ wind field setup conditions at 0.5 seconds time scale. The red colour contours indicate stronger velocity winds up to average $6 \mathrm{~m} / \mathrm{s}$ than the light blue colour depict an average $4 \mathrm{~m} / \mathrm{s}$ of wind speeds [Figures 17, 18 and 19].

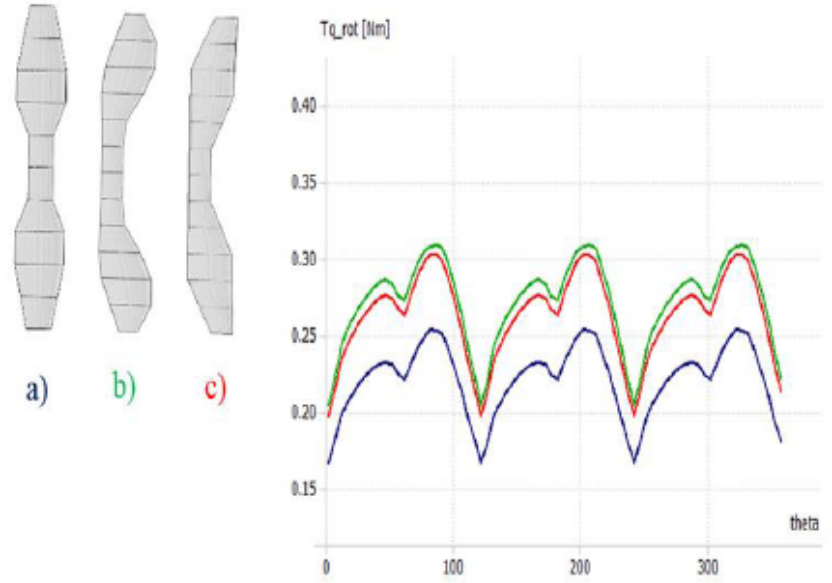

Figure 15: (a) Bowtie blade, (b) Maple seed leaf blade and (c) wing blade shown with colours to indicate in graph (left). Rotational torque (Tq_rot) vs angle of rotation of the blades shown (right).

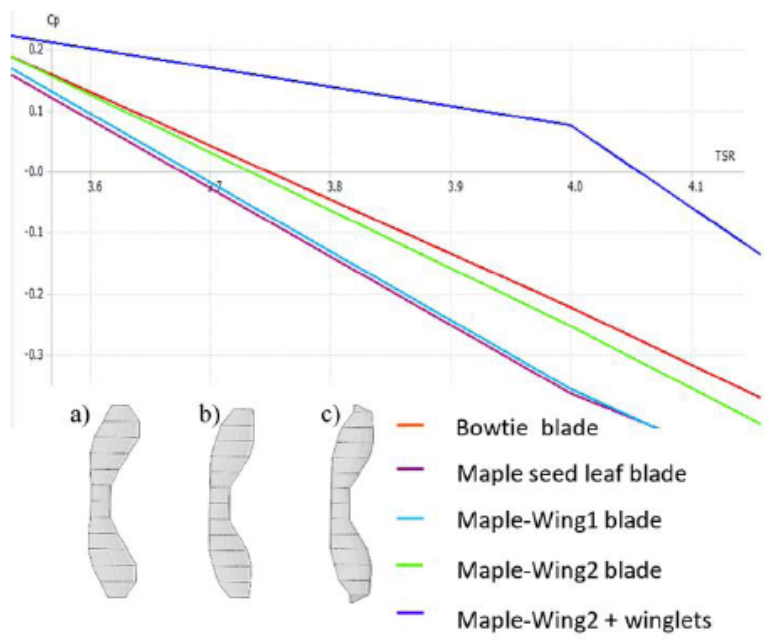

Figure 16: $\mathrm{Cp}$ vs TSR of all the modified blades including blades mentioned in Fig.15 Left bottom: - Maple-wing1 (a), Maple-wing2 (b), Maple-wing2 with winglets (c).

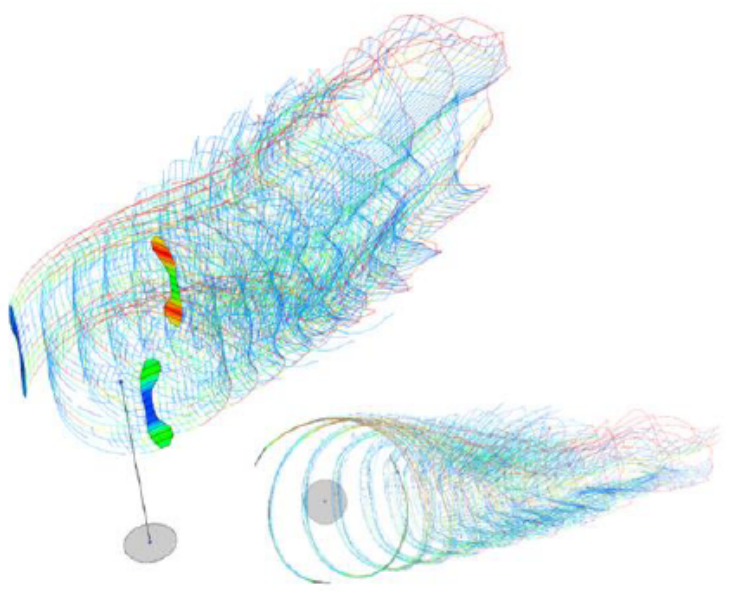

Figure 17: Flow vorticity structure of Maple-wing1 - isometric view (left) and top view (right bottom).

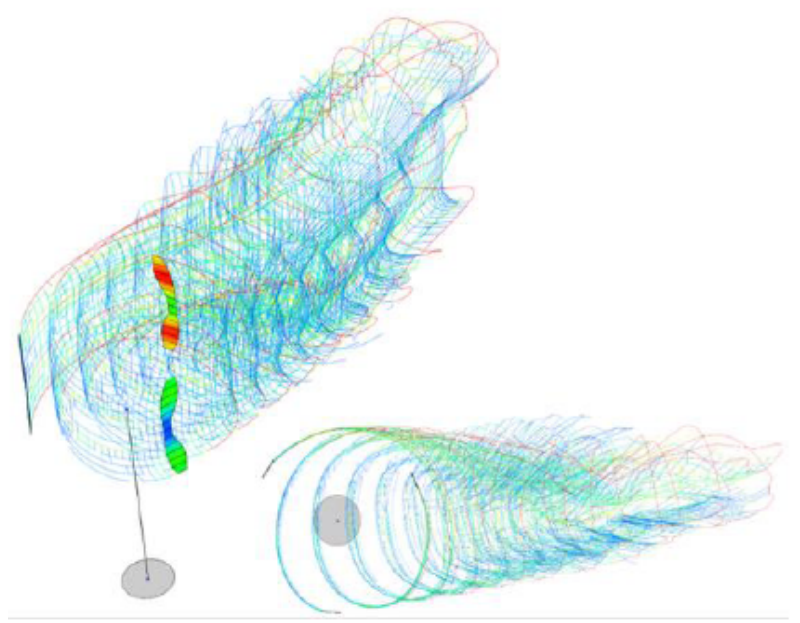

Figure 18: Flow vorticity structure of Maple-wing2 - isometric view (left) and top view (right bottom). 


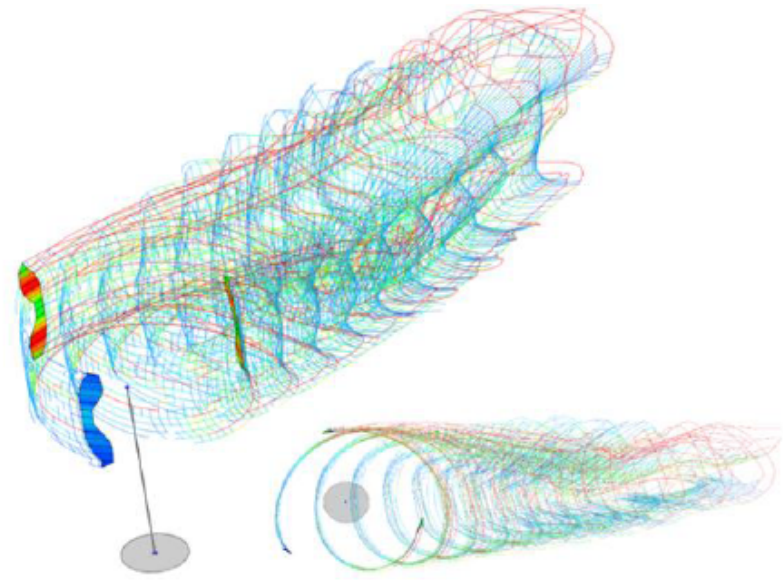

Figure 19: Flow vorticity structure of Maple-wing2 with winglets - isometric view (left) and top view (right bottom).

\section{DISCUSSIONS}

The tests and simulations in this paper are based on the findings of three major design requirements of an effective turbine blade through bio-features under turbulent low wind speed condition for factors such as lift-drag, rotor power, tip-speed and rotor torque which are compared and discussed based on the findings below in the subsequent sections.

\subsection{Foil comparison}

The FX 63-137 foil due to its negative camber and non-symmetricity there is higher $\mathrm{Cp}$ value (coefficient of pressure) with more even pressure distribution around the foil vicinity under required circular blade path. FX 63-137 also gave a thicker boundary layer with faster lift due to smaller lift angle [Figure 4]. The corrugated FX 63-137 foil cross section blades showed good lift characteristic $C_{l}$ [Figure 8] and average $C_{p}$ value (coefficient of power) but did not show improvements in terms of torque coefficient [Table 4], But compared to foils such as FX 63-137, wing tail and modified corrugate-wing foil, the corrugated foil showed improvement in coefficient of performance by $65.1 \%$ over the initial FX 63-137 blade [Table 4] [7, 14]. The FX foils from the paper are compared with the XFOIL simulation results of $F X$ and corrugated foils [Figure 20], The peak values have improved in the Corrugated foil in terms lift coefficient with a much lower drag coefficient when compared to the FX 63-137 foil from [10] as well as the interpolated FX 63-137 foil.

\subsection{Comparison of Aspect Ratio}

Height to radius ratio close to 1 was an ideal range as shown in [Figure 9] with $0.2 \mathrm{~m}$ chord length over
$0.4 \mathrm{~m}$ showed slight improvement as well. The smaller chord with larger radius provided more torque which increased the $\mathrm{Cp}$ value. Turbine structure with $H / R$ equal to 1 with chord length $20 \mathrm{~cm}$ showed an efficiency improvement of $36.8 \%$ over a turbine structure with $H / R$ equal to 0.4 with a larger chord length of $40 \mathrm{~cm}$ [1]. Inward blade orientation was important in controlling the turbulent vortex distribution to enable better wake visible in the LLT [Figure 10]. From the DMS simulations the inward showed better power coefficient at higher tip speed range with $25.9 \%$ increase in $\mathrm{Cp}$ at $\mathrm{TSR}=4$ [Figure 11] and also an increase in momentary power at low velocities with $40.1 \%$ increase in power at an ideal wind speed of $5 \mathrm{~m} / \mathrm{s}$ [Figure 12].

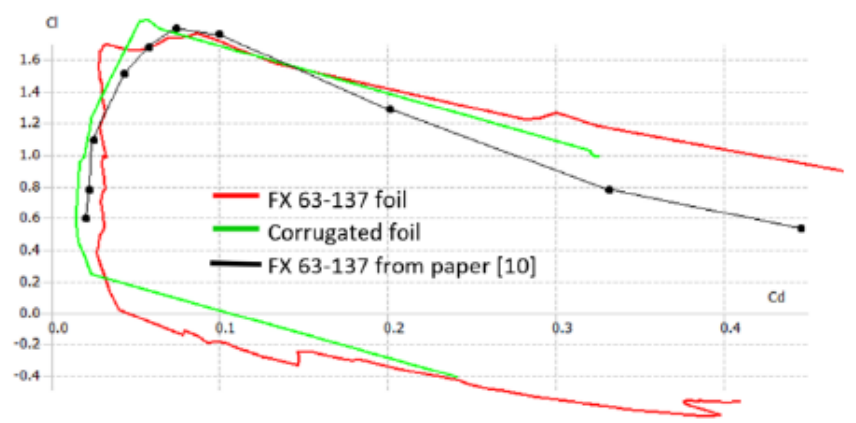

Figure 20: Comparison and validation of base foils and modified foils [10].

\subsection{Blade Comparison}

The proposed bowtie blade's narrow centre-edge provided lesser vortex accumulation at the back [Figure 13]. The narrow edge reduced blade bending stress at the edges enabling smoother edge path [Figure 13 (b)] and it improved the effective TSR range up to 6 [Figure 14] With centre-edge design as basis the further modifications with combination of Maple seed leaf and flight wing showed better rotational torque improvement by $23.52 \%$ in Maple leaf blade and by $20.58 \%$ in wing blade when compared to Bowtie [Figure 15] with optimized gradual varying chord lengths across the height of the blade the $\mathrm{Cp}$ value of the Maple-wing2 [Figure 16 (b)] has improved over the Maple seed leaf blade [Figure 15 (b)] by over $16.6 \%$ and the addition of edge winglet tips to Maple-wing2 also improved the $\mathrm{Cp}$ by $51.5 \%$ over the blade without the winglets [Figure 16]. The Maple-wing LLT flow structures showed that at equal time intervals the blade winglets improved the number of rotations per second and strength of the wake produced causing the turbulence in the vorticity contours behind the blade after the cut-in positions [Figure 17 and 18] [7]. Also, the flow vorticity structures in Maple-wing2 with winglets shows an increase in the 
strength of winds near the edges with dense boundary layer and lower flow separation vortexes [Figure 19]. From the FX-63 137 foil used in [Figure 20] a straight blade of the same dimensions was compared with corrugated foil straight blade and Maple-wing2 with winglets blade to know the difference in $C_{p}$ values for small TSR shown in [Figure 21]. The Maple-wing2 with winglets showed $15.7 \%$ improvement in terms of coefficient of power and $37.4 \%$ in terms rotational torque over FX 63-137 foil straight blade at a TSR of 4 and average wind speed of $5 \mathrm{~m} / \mathrm{s}$ using rotor and turbine DMS simulations [Table 5] from [Figure 21]. All the values and figures found using verified methods and were compared from the chosen papers and successfully validated with necessary references.

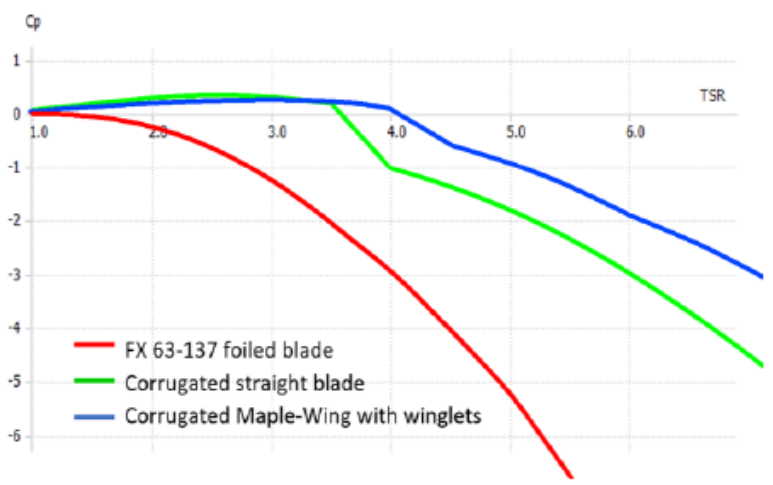

Figure 21: Comparison Cp vs TSR of FX 63-137 straight blade, corrugated straight blade and maple-wing2 with winglets blade.

\section{CONCLUSION}

The simulations under XFOIL, rotor-turbine DMS and LLT under Q-blade software of the Maple-Wing blade plus winglet edge tips with cross-section of a Corrugated Dragon vein FX 63-137 foil showed an overall performance improvement and had the characteristics to provide better power and torque output under dynamic wind speed ranges with better efficiency [7, 9]. The biomimicry implementation over the foil and blade also proved beneficial in maintaining low-stress density and simplistic design, these advantages can be leveraged in large scale wind energy projects such as wind farms and harnessing oceanic winds as well as an energy supply alternative for small scale household appliances and even commercial equipment as a secondary energy source.

It still has the limitations of inefficient self-start, mechanical losses and turbulent wake that could inhibit the actual performance of the VAWT, these require further research with proper experimentation under a wind tunnel setup which is not discussed in this paper.
Table 5: Comparison of Modified Blade Performance Values Derived from Rotor and Turbine DMS Simulations at $5 \mathrm{~m} / \mathrm{s}$ wind Speed Condition

\begin{tabular}{|c|c|c|c|}
\hline Modified Blades Designs & $\mathbf{C}_{\mathrm{p}}$ & $\begin{array}{c}\text { Rotor Torque } \\
\mathbf{( N m )}\end{array}$ & Power (W) \\
\hline \hline Bowtie blade & -0.21 & 0.395 & 12.1 \\
\hline Maple seed leaf blade & -0.36 & 0.376 & 10.4 \\
\hline Wing blade & -0.34 & 0.355 & 9.3 \\
\hline Maple-wing1 & -0.30 & 0.371 & 9.5 \\
\hline Maple-wing2 & -0.25 & 0.383 & 10.5 \\
\hline Maple-wing2+winglets & 0.09 & 0.443 & 11.4 \\
\hline
\end{tabular}

The final proposed Corrugated FX foil embedded Maple-wing blade with added back edge winglets has shown to be a potential VAWT blade over FX-63137 foil straight blade with a $21 \%$ power efficiency improvement and a $39 \%$ rotary torque improvement calculated using DMS simulations at a low wind speed of $5 \mathrm{~m} / \mathrm{s}$ and TSR at 4 . The extracted data provided in this paper is matching well with the reference data from the software used and specified.

\section{REFERENCES}

[1] Hand B, Kelly G and Cashman A. "Aerodynamic design and performance parameters of a lift-type vertical axis wind turbine: A comprehensive review", Renewable and Sustainable Energy Reviews, 2021; 139: 110699, 13640321. https://doi.org/10.1016/j.rser.2020.110699

[2] Tjiu W, Marnoto T, Mat S, Ruslan MH and Sopian K "Darrieus vertical axis wind turbine for power generation I: Assessment of Darrieus VAWT configurations", Renewable Energy, 2015; 75: 50-67. https://doi.org/10.1016/j.renene.2014.09.038

[3] Battisti L, Brighenti A, Benini E, Castelli M. "Analysis of Different Blade Architectures on small VAWT Performance". Journal of Physics: Conference Series. 2016; 753: 062009. https://doi.org/10.1088/1742-6596/753/6/062009

[4] Islam M, Ting D, Fartaj A. "Desirable Airfoil Features for Smaller-Capacity Straight-Bladed VAWT". Wind Engineering. 2007; 31: 165-196.

https://doi.org/10.1260/030952407781998800

[5] Anweiler S, Fedak W, Gancarski W, Ulbrich R. "Determination of the number of VAWT blades based on power spectrum". Journal of Mechanical and Energy Engineering, 2017; 1.

[6] Cai X, Zhang $Y$, Ding W, Bian S. "The aerodynamic performance of H-type Darrieus VAWT rotor with and without winglets: CFD simulations". Energy Sources, Part A: Recovery, Utilization, and Environmental Effects. 2019; 1-12. https://doi.org/10.1080/15567036.2019.1691286 
[7] Seidel C, Jayaram S, Kunkel L. Mackowski A Structural Analysis of Biologically Inspired Small Wind Turbine Blades. Int J Mech Mater Eng 2017; 12: 19. https://doi.org/10.1186/s40712-017-0085-3

[8] Tang D, Fan Z, Lei M, Lv B, Yu L and Cui H. "A combined aerofoil with secondary feather inspired by the golden eagle and its influences on the aerodynamics," International Journal of Green Energy, Chin. Phys. 2019; B 28(3): 034702. https://doi.org/10.1088/1674-1056/28/3/034702

[9] Mulligan R. "Bio-inspired aerofoils for small wind turbines," Renewable Energy and Power Quality Journal (RE\&PQJ) ISSN 2172-038 X,18, 2020. https://doi.org/10.24084/repqj18.488

[10] Meana-Fernández A, Díaz-Artos L, Oro J MF, VelardeSuárez S. "An Optimized Airfoil Geometry for Vertical-Axis Wind Turbine Applications". I J green Energy, 2020; 17(3): 181-195 1464 https://doi.org/10.1080/15435075.2020.1712211
[11] Alaskari $\mathrm{M}$, Abdullah $\mathrm{O}$, Majeed $\mathrm{MH}$. "Analysis of Wind Turbine Using Q-Blade Software". IOP Conference Series: Materials Science and Engineering, 2019; 518: 3. https://doi.org/10.1088/1757-899X/518/3/032020

[12] Akhmedov D. Sh, Eryomin DI, Yagfarova NI, Turarbekov MK "Mathematical Model To Calculate The Performance Of Low Power Vertical Axis Wind Turbine", International Journal of Advances in Science, Engineering and Technology 2016; 4(1): 23-25.

[13] Momeni H, Domagała M. "CFD simulations of hydrofoils for tidal turbines". Czasopismo Techniczne, 2017; 10: 193-199.

[14] Anand S, Pandey A, Sharma A, Kini CR, Effect of trailing edge roundness on FX 63-137 and Selig S1223 Airfoil, ARPN Journal of Engineering and Applied Sciences. 2017; 12.

DOI: https://doi.org/10.31875/2409-9848.2021.08.1

(C) 2021 Prathik et al.; Zeal Press.

This is an open access article licensed under the terms of the Creative Commons Attribution Non-Commercial License

(http://creativecommons.org/licenses/by-nc/3.0/), which permits unrestricted, non-commercial use, distribution and reproduction in any medium, provided the work is properly cited. 